MATEC Web of Conferences 42, 03014 (2016)

DOI: $10.1051 /$ matecconf/20164203014

(C) Owned by the authors, published by EDP Sciences, 2016

\title{
Paper-based Pneumatic Locomotive Robot with Sticky Actuator
}

\author{
Xiaohan $\mathrm{Du}^{1}$, Hongtao $\mathrm{Wu}^{2}$, Jie $\mathrm{Qi}^{3}$, Shin Young Jeong ${ }^{1}$, Feng $\mathrm{Ni}^{1}$ \\ ${ }^{1}$ HKUST, Department of Mechanical and Aerospace Engineering, Kowloon, Hong Kong \\ ${ }^{2}$ Beihang University, School of Energy and Power Engineering, Beijing, China \\ ${ }^{3}$ HKUST, Department of Physics, Kowloon, Hong Kong
}

\begin{abstract}
Demands for small-scale and low-cost robots have witnessed a great increase in recent years [1-5]. This paper introduces the design and fabrication of a novel, simple, low-cost and designer-friendly locomotive robot. The materials and tools to build the robot originate from everyday life. The robot is pneumatically powered and manually controlled by simply pumping and vacuuming the syringe repeatedly, which realizes reliable locomotion by folding and opening of the planes. In order to realize this complicated motion, a "3D Sticky Actuator" is developed. The motion and force analysis of actuator are then modelled by the numerical method to develop the relations between design parameters. This suggests a systematic and user interactive way of manufacturing various shapes of the actuator, depending on user-defined road condition (e.g. obstacles and slopes) and other constraints. One key advantage of the paper-based robot is suggested by its high feasibility.
\end{abstract}

\section{Introduction}

There has been an increasing demand for locomotive robots that can access places where humans are not able to enter. For narrow and small spaces, a small and reliable robot is preferred. Discrete process such as folding process is also favourable under harsh and unpredictable situations. Since continuous motions like wheel rolling and board taxing require complicated systems to control the motion to adjust to the environment, while discrete motion can adapt to the environment change for its simple and ready control. Various folding-based robots have shown estimable accomplishments and potentials in the small-scaled locomotion robot field. RoACH, developed by Hoover et al., is a $2.4 \mathrm{~g}$ robot, realizing its autonomous motion by folding and assembling process [3]. Onal et al. achieved worm-like peristaltic locomotion by fabricating a $2 \mathrm{D}$ flat sheet into a 3D complex folding patterning [4]. Miyashita et al. realized the self-pop-up motion of a cylindrical structure by global heating [5]. As robots enter our life, demands for rapid fabrication and reliability to tackle unpredictable situations have seen a great increase. For the sites where the robots perform their task are already structured, fixed-architecture robots may perform well; for sites where the robots operate are unstructured, selfreconfiguring robots, reconfiguring different modules of its body, are more likely to confront this challenge more successfully [5-7].

Inspired by the folding and opening mechanism of Chinese hand-held fan in Figure 1, we introduce a paperbased locomotive robot (Figure 2), which accomplishes its reconfiguring folding process pneumatically with tangible actuators. The robot features not only low-cost, but also readily manufactured. Paper, membranes, tubes, syringes, soldering iron, scissors are used for manufacturing the robot. These materials and tools can be found ubiquitously in almost everyone's daily life. The key component, the 3D Sticky Actuator, can change its shapes when inflated and vacuumed. The idea of this novel actuator is developed from Niiyama's Sticky Actuator [8].

This paper contributes to the detailed design and fabrication of the robot and the 3D Sticky Actuator. A numerical modelling is employed to analysis the motion and force acting on the robot during operation. Road tests of the robot under slopes and obstacles conditions are then demonstrated.

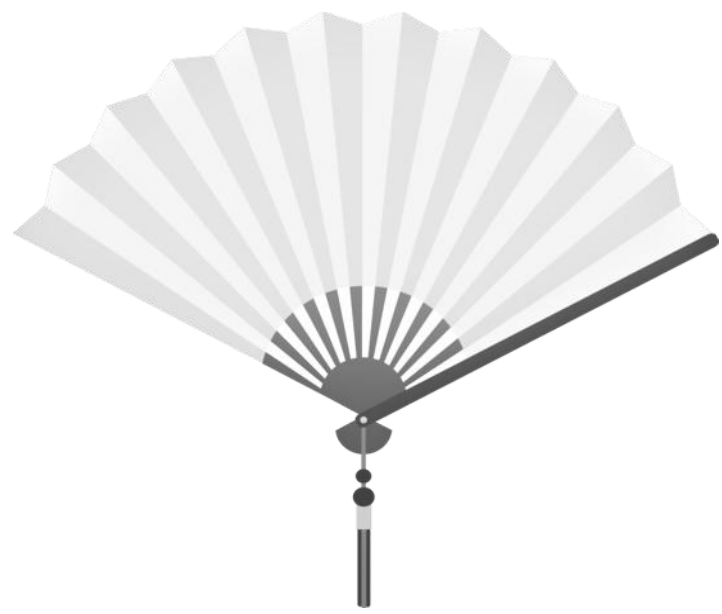

Figure 1. A Chinese hand-held fan [9].

a Hongtao Wu: hongtao_wu@buaa.edu.cn, ${ }^{\mathrm{b}}$ Xiaohan Du: xduab@connect.ust.hk 


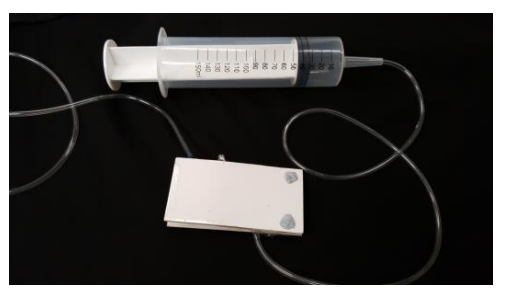

Figure 2. The paper-based pneumatic locomotive robot.

\section{Design of the robot}

The idea of robot's design comes from the movement of the traditional fan, folding and unfolding, featuring reconfiguration. But the connection of layers of the robot is analogous to the snake-like route, contrasting to the fan. To achieve the reconfiguration, pneumatic powered actuators are used.

\subsection{Design}

The robot is composed of three separate cardboards joined by tape at joints, each measuring $3 \mathrm{~cm}$ by $10 \mathrm{~cm}$. Two HK dollar coins are used as the counterbalanced weight at the edge of the cardboards. Figure 3 and 4 show the folded and unfolded status from side view. When the air is not injected in, the robot is folded in a compressed Z-shape. Between these layers, 3D actuators are inserted. When air is injected in, the 3D actuator extends with a wide opening angle. For each actuator, a tube is connected to a $150 \mathrm{ml}$ syringe.

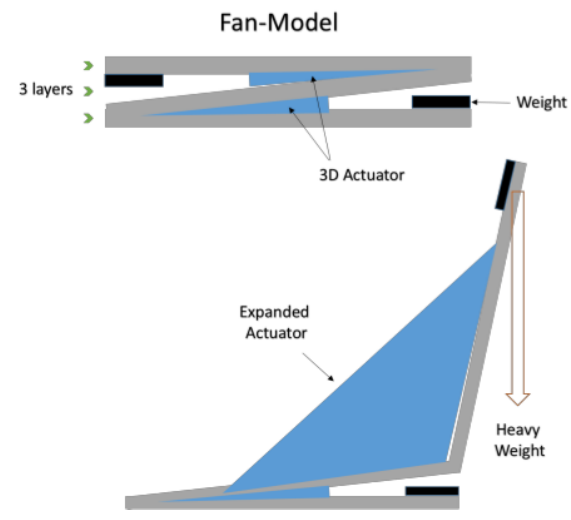

Figure 3. The folded and unfolded status.
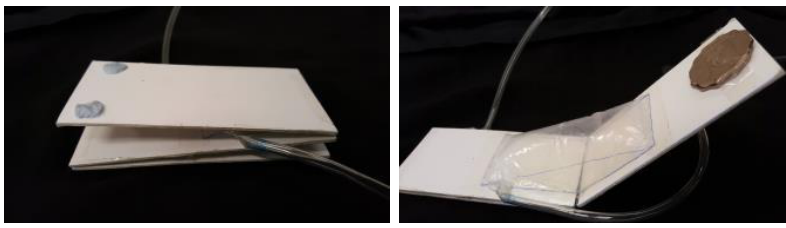

Figure 4. The side view of the robot in folded and unfolded status.

\subsection{Design}

The mechanism for the locomotion of the paper-based fan model robot is shown in Figure 5. State 1 is when the air is not injected in both actuators. The three layers of "fan plane" are folded. Air is then injected gradually into the actuator and the top layer rotates $(1 \rightarrow 2)$. The angle between the top and the middle layer is 125 degrees (state 2 ). There is a counterbalance weight placed at the edge of the board, producing a gravitational moment for the whole system of robot to rotate. This status is shown in state 3. After the whole robot rotates, air is ejected from the actuator, pulling the lateral two planes to fold $(3 \rightarrow 1)$. The robot returns to state 1 but has rotated 180 degrees and moved a distance equal to the length of one board theoretically. After one cycle, the positions of the actuator switch. The top actuator, which has not been used during the previous process, becomes active and begins the whole process again. This process becomes continuous, allowing to robot to move forward.

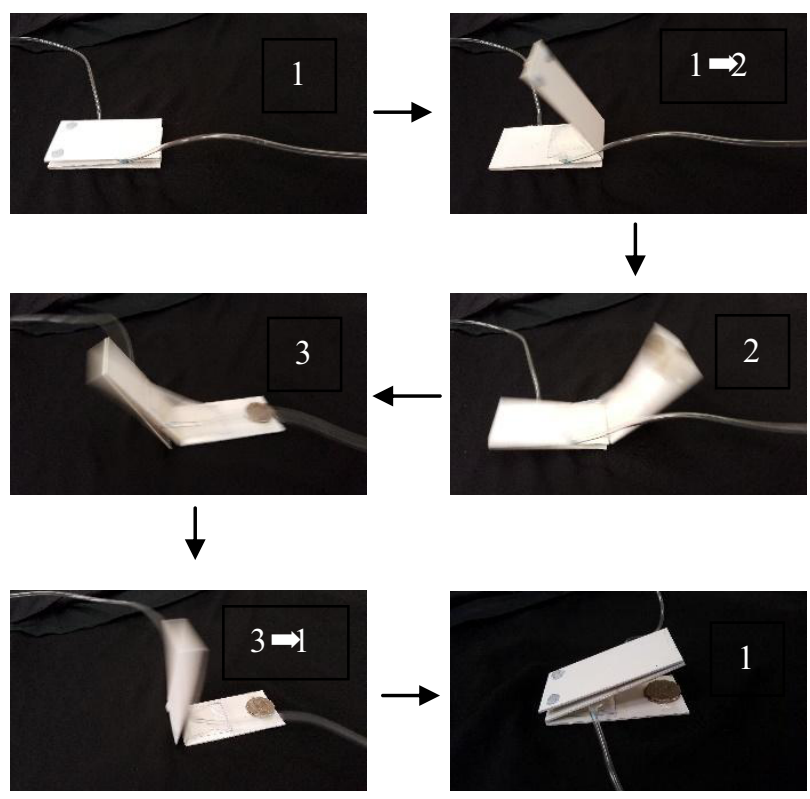

Figure 5. A cycle of the robot movement.

\section{The 3D sticky actuator}

The key part of the locomotion is to move the centroid of the robot forward. This motion is achieved by deforming the robot into specific shapes and it relies on the gravity moment to move the centroid. The original "Sticky Actuator" shown in Figure 6 deforms its shape by pumping and vacuuming air into and out of the actuator [8]. Despite its reliability, it can only apply valid forces in one direction. So a "3D Sticky Actuator" of a prism shape with a triangular sectional area is proposed to achieve the two- directions, namely, forward and backward force.

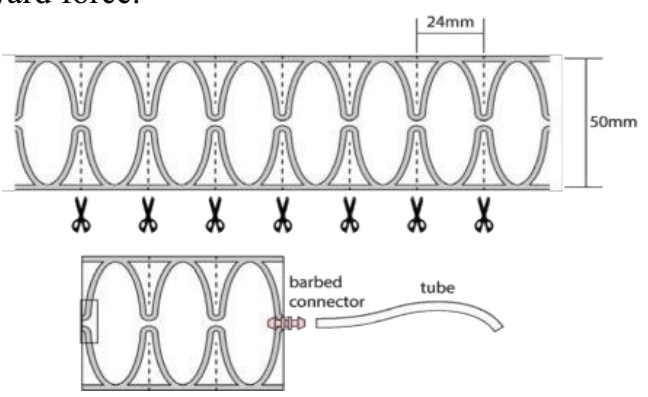

Figure 6. Sticky Actuator [8]. 


\subsection{Design}

The design of the prism 3D sticky actuator is shown in Figure 7. The prism 3D actuator is a triangular prism and is compatible to the shape of the robot. Two sticky surfaces $(3.5 \mathrm{~cm}$ by $3 \mathrm{~cm})$ are adhered to the cardboard layers of the robot. When the actuator is gradually inflated with air, the surface not attached to the cardboard $(6.25 \mathrm{~cm}$ by $3 \mathrm{~cm})$ will be stretched. The three angles of the sectional triangle are $125,27.5$, and 27.5 degrees respectively for the level ground condition. For different road conditions, such as slope and obstacle, the designs of the actuator are different.

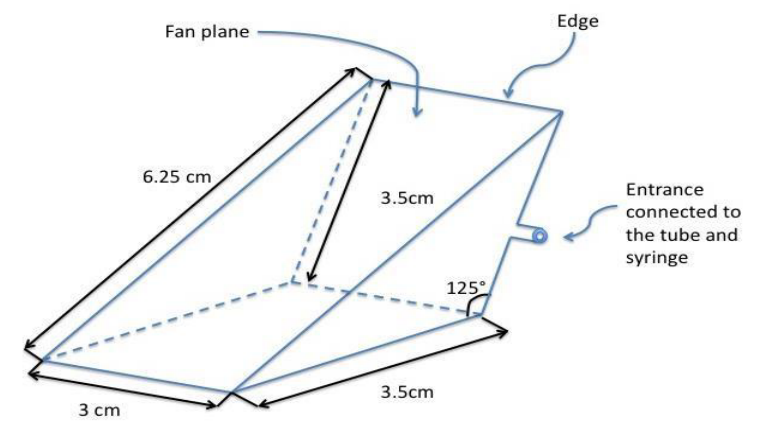

Figure 7. Design of the 3D sticky actuator.

\subsection{Fabrication}

The fabrication process of the "3D Sticky Actuator" is similar but more complicated compared to the "Sticky Actuator" [8]. The original "Sticky Actuator" is made of two identical shapes of layers of membrane sealed together. But for "3D Sticky Actuator", the number of surfaces is more than that of the $2 \mathrm{D}$ version. The fabrication procedure is as follow. Firstly, draw the unfolded shape of the 3D actuator. Secondly, select the edge that needs to be sealed and fold the corresponding edges of the plane shape together. In order to stabilize the membrane when sealing, tape the membrane to the nonstick bakery paper. Thirdly, seal the edge with the soldering iron. The hot iron will melt the membrane and seal the edges together. Fourthly, repeat the above process and form a closed actuator.

\section{Mathematical model 3D sticky actuator}

A mathematical model (Figure 8) is built to analyze the force applied by the actuator and simulate the motions of the robot when the dimensions are given. The results are obtained by numerical method in MATLAB. The purpose of developing physical analysis is to ease the design process of users, making the robot designer-friendly. The motions of the robot are described as follow:

1. Opening motion: Air is injected; the top layer rotates about the pivot $\mathrm{O}$ (as shown in Figure 8).

2. Transmitting motion: After the actuator is fully inflated, the lying layers are pulled up, and the whole robot rotates about the pivot $\mathrm{O}$.
3. Folding motion: After the top layer strikes the ground, the air is vacuumed from the actuator, pulling the two folded layers to the ground.

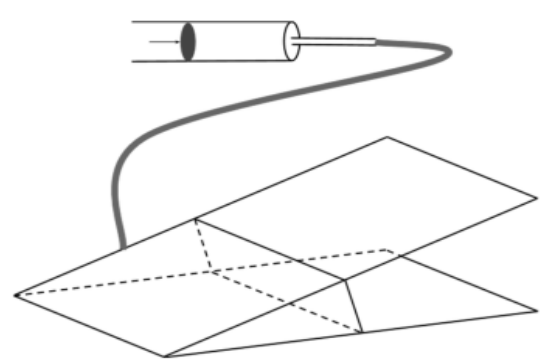

Figure 8. Mathematical model for the robot.

\subsection{Assumptions for the model}

1. There is no leakage of gas during the whole process.

2. The gas is considered ideal, and any process is quasistatic process.

3. When the gas is not completely injected into the actuator, the shape of the actuator is considered a triangular prism.

4. The velocity of the pumping of syringe is constant.

5. Air friction and mechanical frictions are ignored.

\subsection{Assumptions for the model}

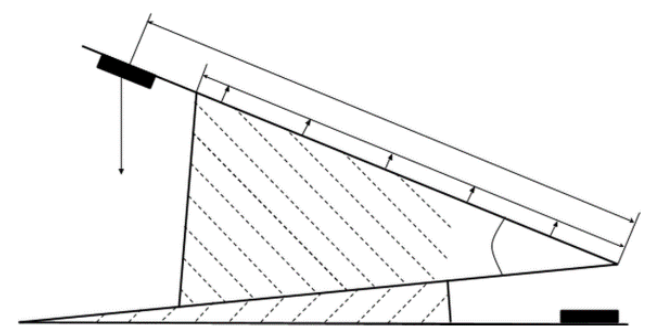

Figure 9. Forces applied on the opening layer.

The force analysis of the robot when in opening motion is shown in Figure 9. Gravitational force of the counterbalance weight is exerted in the vertical direction. The mass of the board is neglected. The pressure force exerted by the gas in the actuator is perpendicular to the board's surface. The equation of moment about the turning pivot $\mathrm{O}$ is,

$$
\frac{1}{2} P(D l) l-G_{c} l_{0} \cos \theta=I_{C} \ddot{\theta}
$$

where the depth of the prism equals to $D ; I_{C}$ is the total moment of inertia of the moving layer. The volume $V$ of the actuator at position $\theta$ can be approximated as the volume of the triangular prism with the vertex angle equals to $\theta$. Therefore,

$V(\theta)=\frac{1}{2} l^{2} \sin \theta D$ 
Then

$P(\theta)=\frac{n(\theta) R T}{V}=\frac{n(\theta) R T}{\frac{1}{2} l^{2} \sin \theta D}$

where $n(\theta)$ is the mole of air in the actuator. The volume of the gas in the syringe $V^{\prime}$ at time $t$ is,

$$
V^{\prime}(t)=V_{0}^{\prime}-v t s
$$

where $V_{0}^{\prime}$ is the volume of the syringe, $v$ is the constant pushing speed and $S$ is the cross section of the syringe. The total mole numbers of gas is constant $n_{0}$. Then $n(\theta)$ can be derived as,

$n(\theta)=n_{0}-\frac{P(\theta) V(t)}{R T}$

Combine (3), (4) and (5) and solve for $P(\theta)$ :

$$
P(\theta)=\frac{2 n_{0} R T}{l^{2} \sin \theta D+2\left(V_{0}^{\prime}-v t s\right)}
$$

Plug (6) into (1):

$$
\frac{n_{0} R T}{\sin \theta+2\left(V_{0}^{\prime}-v t s\right) / D l^{2}}-G_{C} l_{0} \cos \theta=I_{C} \theta
$$

\subsection{Transmitting motion}

After the air is fully injected in, the entire robot rotates around pivot $\mathrm{O}$ under the moment by the weight of the coin. The equation is

$$
-G_{c} l_{0} \cos \theta=I_{C} \ddot{\theta}
$$

The transmitting motion will end when $\theta=\pi$.

\subsection{Folding motion}

Since the weight of the cardboard is neglected, the folding motion is simplified as a motion with constant velocity $\omega$. The total volume of the syringe and actuator is assumed to be constant. Thus,

$\frac{\Delta\left(\frac{1}{2} l^{2} D \sin \theta\right)}{\Delta \mathrm{t}}=v S$

Approximate $\Delta \sin (\theta)$ by $\theta$,

$\omega=\frac{205}{12 D}$

\subsection{Simulation results}

Combine (7), (8) and (10), the motion of the actuator and cardboard can be obtained. Figure 10 and 11 demonstrate the angular position and velocity diagram plotted in MATLAB. The simulated total time for the robot to complete a cycle is $0.5146 \mathrm{~s}$.

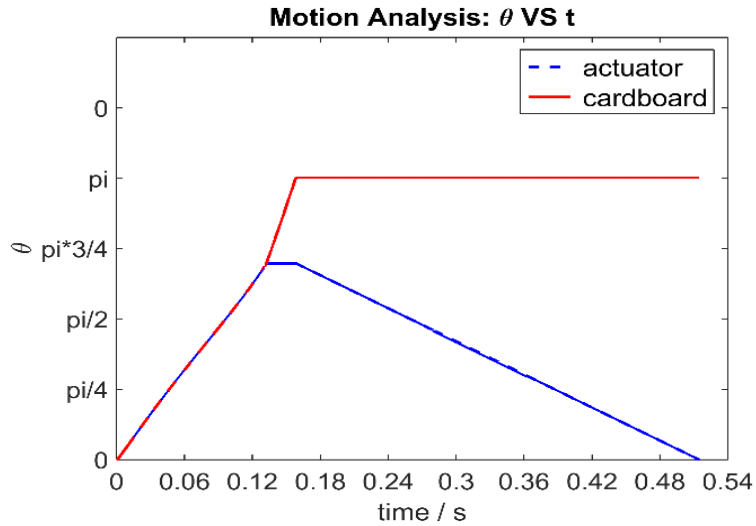

Figure 10. Simulated angular position diagram.

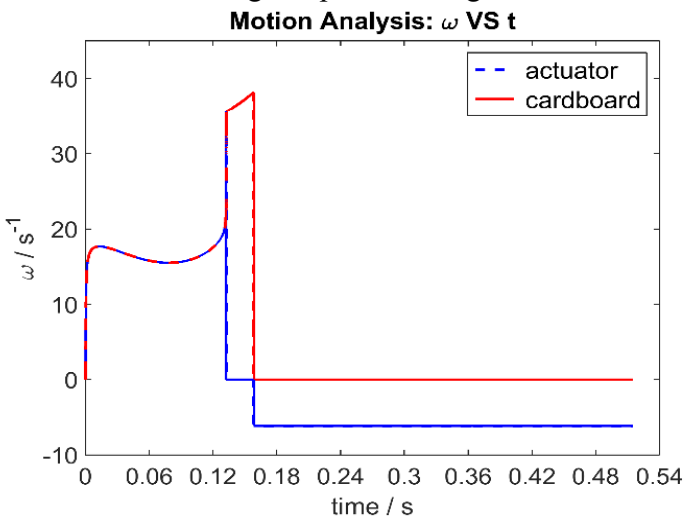

Figure 11. Simulated angular velocity diagram.

\subsection{Slope condition}

The presented locomotive robot is capable to operate under different road conditions, e.g. slopes and obstacles. The following model shows details of its motions.

A critical climbing angle is defined to characterize the robot's performance on inclined surface. The model only considers the gravitational torque, namely ignoring the sliding effect on the inclined surface.

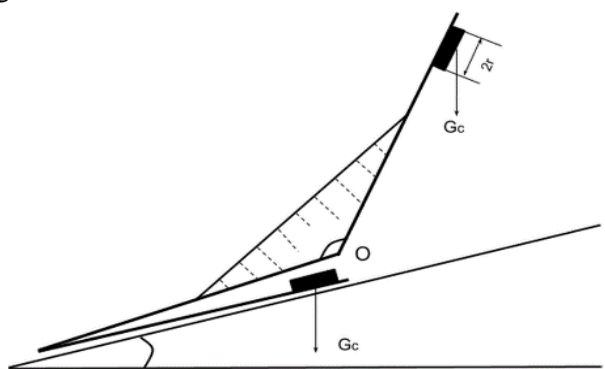

Figure 12. Static critical angle.

The critical condition for robot to proceed to transition motion after opening is shown in Figure 13. The equilibrium equation is

$$
G_{c} r=G_{c}\left(l_{0}-r\right) \cos (\pi-\varphi+\alpha)
$$

in which $\mathrm{r}$ is the radius of the coin; $\varphi$ is the maximum opening angle of the actuator; $\alpha$ is the static critical slope angle.

When the robot is in motion, there would be rotational momentum, which yields a smaller critical slope angle 
depending on the angular velocity of the board. Therefore, the robot is able to climb up the slope with angle smaller than $\alpha$, and may climb up the slope with angle larger than $\alpha$.

For the presented prototype, the calculated static critical slope angle is 25.6 degrees.

\section{Test on performance}

To verify the modelling result, velocity tests and slope tests are conducted.

\subsection{Speed test}

The speed test was practiced 5 times along the distance of $1 \mathrm{~m}$ for each robot. Time for travelling is measured and speed of robot is calculated. The data for each robot are measured and organized in Table I.

Table 1. Result of speed test

\begin{tabular}{|c|c|c|}
\hline Order & Time $(\mathrm{s})$ & Speed $(\mathrm{cm} / \mathrm{s})$ \\
\hline 1 & 9 & 11.1 \\
\hline 2 & 11 & 9.9 \\
\hline 3 & 10 & 10.0 \\
\hline 4 & 10 & 10.0 \\
\hline 5 & 10 & 10.0 \\
\hline Mean & 10 & 10.0 \\
\hline
\end{tabular}

The mean time for the injection/exhaustion process is $0.5 \mathrm{~s}$, which matches the simulation result very well.

\subsection{Slope test}

The prototype is also tested under condition of slope with different variation. Frictional material was added on the cardboard surface to exclude the sliding. The result is shown in Table II below.

Table 2. Result of slope test

\begin{tabular}{|c|c|}
\hline Slope Angle (degree) & Performance \\
\hline 15 & Climb steadily \\
\hline 20 & Climb steadily \\
\hline 25 & Climb steadily \\
\hline 30 & Fail to climb \\
\hline
\end{tabular}

The result in the table is consistent with the calculation result ( $a=25.6$ degrees). Therefore, the mathematical model is valid.

\section{Conclusion}

In this paper, we proposed a paper-based pneumatic locomotive robot based on the mechanism of the opening and folding mechanism of Chinese hand-held fan. In order to achieve the special mechanism, a "3D Sticky Actuator" is developed. The actuator can achieve twodimensional actuating, compared to Niiyama's "Sticky
Actuators". It can work properly with any pneumatic power. For different track condition, the best dimensions are different and need to be carefully scrutinized when designing. Therefore, a physical model and a numerical computing program are developed to help users to customize their design by simply inputting their design requirements. The high feasibility in material and manufacturing method, also ensure the efficiency in human-robot interaction. Future studies are focused on developing an untethered model. By installing Arduino, pump, solenoid valve and batter, the present tethered model can be updated. The untethered model is expected to be operated without direct human interaction.

\section{Acknowledgement}

The authors wish to thank Prof. Yi-Kuen Lee and Mr. Feng Ni for their support and precious advices.

\section{References}

1. S. Miyashita, S. Guitron, M. Ludersdorfer, C. R. Sung, and D. Rus, "An untethered miniature origami robot that self-folds, walks, swims and degrades," in IEEE International Conference on Robotics and Automation (ICRA), 2015.

2. S. Miyashita, L. Meeker, M. Göldi, Y.Kawahara, and D. Rus, "Self-folding printable elastic electric devices: resistor, capacitor and inductor," in IEEE International Conference on Robotics and Automation (ICRA), 2014, pp. 1446-1449.

3. A. M. Hoover, E. Steltz, and R. S. Fearing, "RoACH: An autonomous $2.4 \mathrm{~g}$ crawling hexapod robot," in IEEE/RSJ International Conference on intelligent Robots and Systems (IROS), 2008, pp. 26-33.

4. C. D. Onal, R. J. Wood, and D. Rus, "Towards printable robotics: Origami-inspired planar fabrication of three-dimensional mechanisms," in IEEE International Conference on Robotics and Automation (ICRA), 2011, pp. 4608-4613.

5. S. Miyashita, C. D. Onal, and D. Rus, "Self-pop-up cylindrical structure by global heating," in IEEE/RSJ International Conference on Intelligent Robots and Systems (IROS), 2013.

6. M. Yim, "New locomotion gaits," in IEEE International Conference on Robotics and Automation (ICRA), 1994, pp. 2508-2514.

7. K. Kotay, D. Rus, M. Vona, and C. McGray, "The self- reconfiguring robotic molecule," in IEEE/RSJ International Conference on Intelli-gent Robots and Systems (IROS), 1998, pp. 424-431.

8. Niiyama R., Sun X., Yao L., Ishii H., Rus D., and Kim S. "Sticky Actuator: Free-Form Planar Actuators for Animated Objects," in International Conference on Tangible, Embedded, and Embodied Interaction (TEI), ACM Press (2015), pp. 77-84.

9. Aragon, Liz. Light Blue Chinese Hand Fan. Digital image. Sweet Clip Art. N.p., 13 Sept. 2013. Web. 19 Oct. 2015. <http://sweetclipart.com/light-bluechinese-hand-fan-1543>. 